\title{
PRIMAL IDEALS AND ISOLATED COMPONENTS IN NONCOMMUTATIVE RINGS ${ }^{1}$
}

\author{
BY \\ WILFRED E. BARNES
}

Introduction. L. Fuchs [2] has given for Noetherian rings a theory of the representation of an ideal as an intersection of primal ideals, the theory being in many ways analogous to the classical Noether theory. An ideal $Q$ is primal if the elements not prime to $Q$ form an ideal, necessarily prime, called the adjoint of $Q$. Primary ideals are necessarily primal, but not conversely. Analogous results have been obtained by Curtis [1] for noncommutative rings with unit element, using a definition of right primal ideal which does not, however, reduce to that of Fuchs in a commutative ring. In this paper an alternative definition of right primal ideal in a general ring is given, which reduces to Fuchs' for commutative rings and to Curtis' for rings with unit element and ascending chain condition (A.C.C.) for ideals. This definition is based on a definition of "not right prime to $A$ " which associates with any ideal $A$ certain maximal not right prime to $A$ ideals, analogous to Krull's maximal associated primes. These maximal not right prime to $A$ ideals apparently are not necessarily prime unless a condition of "uniformity," which is weaker than the A.C.C., is imposed. In $\$ 3$ a discussion of primal decompositions in rings without finiteness conditions is given, and in $\$ 4$ the FuchsCurtis decomposition theorems are obtained for rings with A.C.C. for ideals. In $\S 5$ a new definition of the right associated primes of an ideal is given, and the maximal such ideals are determined. Following the methods of Murdoch [6], upper and lower right isolated $B$-components of an ideal $A$, where $B$ is any divisor of $A$, are defined and their properties investigated in $\S 6$. If $B$ is a maximal not right prime to $A$ ideal, the isolated $B$-components of $A$ are called upper and lower principal component ideals of $A$, and reduce to Krull's principal component ideals in commutative rings. It is shown in $\$ 7$ that under certain conditions an ideal is the intersection of its upper principal components, and that any ideal in an associative ring is the intersection of its lower principal components.

1. Notation and definitions. We shall use $R$ to denote an associative ring which will be noncommutative unless otherwise specified. The term ideal will always mean two-sided ideal. Proper ideals in $R$ will be denoted by $A, B, \ldots$

Presented to the Society, June 19, 1954; received by the editors July 6, 1954.

(1) The material for this paper is taken from the author's Ph.D. thesis, written under the direction of Professor D. C. Murdoch at the University of British Columbia. 
and elements of $R$ by $a, b, x, y, \cdots$. The symbols $\sum A_{\alpha}$ and $A+B$ will denote ideal sums of ideals, and $\cup A_{\alpha}$ and $A \cup B$ will denote set theoretic unions. By $\{A \mid A$ has property $P\}$ and $\{x \mid x$ has property $P\}$ we shall mean respectively the set of ideals having property $P$ and the set of elements having property $P$. The symbol $(x)$ will mean the principal ideal generated by $x$ and $(x, y, \cdots)$ the ideal generated by $x, y, \cdots$.

Definition 1. $A x^{-1}=\{y \mid y R x \subseteq A\}$ and $A B^{-1}=\{y \mid y R B \subseteq A\}$. Evidently both $A x^{-1}$ and $A B^{-1}$ are ideals and both contain the ideal $A$.

Definition 2. The element $x$ is not right prime (nrp) to $A$ if $A x^{-1} \neq A$. Otherwise $x$ is right prime (rp) to $A$.

Definition 3 . The ideal $B$ is nrp to $A$ if every $b$ in $B$ is nrp to $A$. Otherwise $B$ is $\operatorname{rp}$ to $A$.

Definition 4. The ideal $A$ is right primal if $\{x \mid x$ is $\operatorname{nrp}$ to $A\}$ is an ideal, which is then termed the adjoint ideal of $A$. Since there are no ideals nrp to the ring $R$, we shall say that $R$ is primal. As we shall consider only right primal ideals, we shall simply say "primal" instead of "right primal."

Definition 5. An ideal $B$ is uniformly nrp (unrp) to $A$ if $A B^{-1} \neq A$, or equivalently if there exists some $y$ not in $A$ such that $y R B \subseteq A$.

Definition 6. A ring is termed (right) uniform if for any two ideals $A$ and $B$, if $B$ is nrp to $A$ it follows that $B$ is unrp to $A$.

We note that $B$ nrp to $A$ in the sense of Curtis [1] implies $B$ is unrp to $A$, if $B$ is unrp to $A$ then $B$ is nrp to $A$, and if $R$ has a unit element then $B$ unrp to $A$ implies $B$ is nrp to $A$ in the sense of Curtis.

Since the ideal sum of an ascending chain of ideals nrp to $A$ is again nrp to $A$, Zorn's lemma assures the existence of ideals which are maximal in the inclusion ordered set of ideals nrp to $A$. Such an ideal will be termed a maximal nrp to $A$ ideal, and thus $A$ is primal if there is only one maximal nrp to $A$ ideal.

If $R$ is commutative, then $x$ nrp to $A$ is equivalent to $x$ not prime to $A$, and thus our definition of primal agrees with that of Fuchs. If $R$ has a unit element then $A$ primal in the sense of Curtis implies $A$ is primal, and if $R$ is a uniform ring with unit element then $A$ primal implies $A$ is primal in the sense of Curtis. That the condition of uniformity is an essential one is shown by an example of Curtis' paper [1] of a nonuniform ring which has a primal ideal that is not primal in his sense.

\section{Primal ideals.}

Definition 7. By a prime ideal we mean an ideal which is prime in the sense of McCoy [5], that is, $P$ is prime if $x R y \subseteq P$ implies $x$ or $y$ is in $P$. McCoy has shown that this is equivalent to the property that if $P$ divides the product of two ideals then $P$ must divide at least one of them.

Definition 8. A maximal prime of an ideal $A$ is an ideal which is maximal in the inclusion ordered set of prime ideal divisors of $A$ which are nrp to $A$.

We note that in the general case there may be no maximal primes of $A$ 
even if it happens that there are prime divisors of $A$ which are nrp to $A$, since the union of an ascending chain of prime ideals is not necessarily prime.

Lemma 1. If $B$ is maximal in the inclusion ordered set of ideals nrp to $A$ and is unrp to $A$ then $B$ is a maximal prime of $A$.

Proof. Let $x R y$ be contained in $B$ and $y$ be not in $B$. Then since $B$ is unrp to $A$ there exists $z$ not in $A$ such that $z R B$ is contained in $A$, hence $z R x R y$ is contained in $A$. Now $B$ a maximal nrp to $A$ ideal implies the existence of some $y^{\prime} \in(y)+B$ such that $y^{\prime}$ is rp to $A$. But then $z R x R y^{\prime}$ is contained in $A$, which implies $z R x$ is contained in $A$. Thus we have $z R[(x)+B] \subseteq A$ and $(x)+B$ is nrp to $A$. Since $B$ is maximal nrp to $A$ it follows that $x$ is in $B$ as required.

Definition 9. An ideal $A$ is strongly irreducible if $A$ cannot be expressed as an intersection, finite or infinite, of proper divisors. $A$ is irreducible if $A$ cannot be expressed as a finite intersection of proper divisors.

Lemma 2. If $A$ is strongly irreducible then every ideal $B$ nrp to $A$ is unrp to $A$.

Proof. Since $B$ is nrp to $A$ we have $A \subset A b^{-1}$ for every $b \in B$. Hence $A \subseteq \bigcap_{b \in B} A b^{-1}$, and since $A$ is strongly irreducible the inclusion must be proper. Thus there exists $x \notin A$ such that $x \in A b^{-1}$ for all $b \in B$. But then $x R B \subseteq A$ and $B$ is unrp to $A$.

Lemma 3. Every irreducible ideal is primal. Every strongly irreducible ideal is primal with prime adjoint.

Proof. Suppose $b_{1}$ and $b_{2}$ are nrp to an irreducible ideal $A$. Then $A b_{1}^{-1} \supset A$ and $A b_{2}^{-1} \supset A$, hence $A \subseteq A b_{1}^{-1} \cap A b_{2}^{-1}$, and since $A$ is irreducible the inclusion must be proper. Hence there exists $x \notin A$ such that $x \in A b_{1}^{-1} \cap A b_{2}{ }^{-1}$. But then $x R\left(b_{1}-b_{2}\right) \subseteq A$ and $b_{1}-b_{2}$ is nrp to $A$. Thus the set of elements nrp to $A$ form an ideal and $A$ is primal. If $A$ is strongly irreducible then, as we have just seen, $A$ is primal. Then by Lemma 2 the adjoint $P$ of $A$ is unrp to $A$, hence by Lemma $1, P$ is a prime ideal.

The following theorem follows at once from Theorem 1.4 of Curtis [1], and Lemma 3.

THEOREM 1. Every ideal is the intersection of its strongly irreducible primal divisors.

Definition 10. If $P$ is a prime ideal divisor of $A$, the (right) upper isolated $P$-component of $A, U(A, P)$, is the intersection of all ideals which contain $A$ and are such that every element not in $P$ is right prime to them. The upper isolated $R$-component of $A$ is defined to be $A$.

This definition has been shown by Murdoch [6] to be equivalent to his definition, except for the case of the upper isolated $R$-component of $A$, which 
in his definition is the ring $R$. As may be readily verified, however, if the (right) lower isolated $R$-component of $A$ is also defined to be $A$, then all results in Murdoch's paper [6] remain valid, the only changes being simplifications in certain theorems where particular cases no longer have to be considered.

Lemma 4. If $A$ is primal with prime adjoint $P$, then $A=U(A, P)$, the upper isolated P-component of $A$.

Proof. By definition $U(A, P)$ is the intersection of all ideals $B$ such that $B \supseteq A$ and if $x \notin P$ then $x$ is rp to $B$. But $A$ is itself such an ideal and the result follows at once.

Theorem 2. Any ideal $A$ is the intersection of its upper isolated $P_{\alpha}$-components, where the $P_{\alpha}$ are the adjoints of the strongly irreducible primal divisors $A_{\alpha}$ of $A$.

Proof. By Theorem 1 we have that $A$ is the intersection of the $A_{\alpha}$, and by Lemma 3 each $P_{\alpha}$ is prime. Thus the theorem is meaningful as stated.

By Lemma 4 we have $A_{\alpha}=U\left(A_{\alpha}, P_{\alpha}\right)$ and Murdoch has shown in [6] that if $P_{\alpha} \supseteq A_{\alpha} \supseteq A$, then $U\left(A_{\alpha}, P_{\alpha}\right) \supseteq U\left(A, P_{\alpha}\right) \supseteq A$. We thus obtain $A=\cap A_{\alpha}$ $=\cap U\left(A_{\alpha}, P_{\alpha}\right) \supseteq \cap U\left(A, P_{\alpha}\right) \supseteq A$, and the equality follows.

\section{3 . Representations by primal ideals.}

DEFINITION 11. A representation

$$
A=A_{1} \cap A_{2} \cap \cdots \cap A_{n}
$$

of an ideal $A$ as the intersection of ideal divisors of $A$ will be called irredundant if no $A_{i}$ contains the intersection of the remaining ones, and reduced if no $A_{i}$ can be replaced by a proper divisor.

Lemma 5. If (1) is a reduced representation of $A$ by primal ideals $A_{i}$ with prime adjoints $P_{i}$, then an ideal $B$ is nrp to $A$ if and only if $B$ is contained in one of the $P_{i}$.

Proof. (i) If $B$ is nrp to $A$, for any $b \in B$ we may find $x_{b} \notin A$ such that $x_{b} R b \subseteq A \subseteq A_{i}$ for all $i$. But $x_{b} \notin A$ implies $x_{b} \notin A_{i}$ for some $i$, hence $b$ is nrp to $A_{i}$ and $b \in P_{i}$ since $A_{i}$ is primal. Since $b$ is arbitrary in $B$, we conclude that $B \subseteq P_{1} \cup P_{2} \cup \ldots \cup P_{n}$. We may suppose the indexing to be such that $B \subseteq P_{1} \cup P_{2} \cup \ldots \cup P_{k}$ but $B$ is not contained in the union of any proper subset of $P_{1}, P_{2}, \cdots, P_{k}$. Then we may choose $p_{i} \in B \cap P_{i}$ such that $p_{i} \notin P_{j}$ for $j \neq i$, for each $i=1,2, \cdots, k$.

If $k=2$, then $p_{1}+p_{2}$ is in $B$, hence in either $P_{1}$ or $P_{2}$, either of which is contrary to the choice of $p_{1}$ and $p_{2}$. Thus $k \neq 2$.

If $k>2$, then $p_{1} R p_{2} \Phi P_{k}$ since neither of $p_{1}, p_{2}$ is in $P_{k}$ and $P_{k}$ is prime. Choose $r_{1}$ so that $p_{1} r_{1} p_{2}=p_{1}^{\prime} p_{2} \notin P_{k}$. Then $p_{1}^{\prime} p_{2} R p_{3} \Phi P_{k}$ and we may choose $r_{2}$ so that $p_{1}^{\prime} p_{2} r_{2} p_{3}=p_{1}^{\prime} p_{2}^{\prime} p_{3} \notin P_{k}$. Continuing in this way we obtain $p=p_{1}^{\prime} p_{2}^{\prime}$ 
$\cdots p_{k-1} \notin P_{k}$. But $b=p+p_{k} \in B$, and $b$ must be in some $P_{i}$ for $i \leqq k$. If $i<k$ then $p_{k} \in P_{i}$ contrary to assumption, and if $i=k$ then $p \in P_{k}$ contrary to assumption. Hence $k \ngtr 2$ and we conclude that $k=1$, and thus $B \subseteq P_{1}$.

(ii) For $p_{1} \in P_{1}$, let $y$ be such that $y \notin A_{1}$ and $y R p_{1} \subseteq A_{1}$. Then $A_{1}^{\prime}=(y)$ $+A_{1} \supset A_{1}$. Since (1) is reduced, there must exist $y^{\prime} \in A_{1}^{\prime} \cap A_{2} \cap \cdots \cap A_{n}$ and such that $y^{\prime} \notin A$. But $y^{\prime} R p_{1} \subseteq(y) R p_{1}+A_{1} R p_{1} \subseteq A_{1}$, hence $y^{\prime} R p_{1} \subseteq A$ and $p_{1}$ is nrp to $A$. But since $p_{1}$ is arbitrary in $P_{1}$ we conclude that $P_{1}$ is nrp to $A$, and similarly the other $P_{i}$ are nrp to $A$.

THEOREM 3. If (1) is a reduced representation of $A$ by primal ideals with prime adjoints $P_{i}$, then the maximal nrp to $A$ ideals are the maximal primes of $A$ and are in fact the maximal elements of the inclusion ordered set $P_{1}, P_{2}, \cdots$, $P_{n}$.

Proof. Suppose $P$ is maximal nrp to $A$, i.e., $P$ is nrp to $A$ and if $Q \supset P$ then $Q$ is rp to $A$. By Lemma $5, P$ is contained in some $P_{i}$. But $P_{i}$ is nrp to $A$ for all $i$ by the other half of Lemma 5 , hence the maximality of $P$ implies that $P=P_{i}$ for some $P_{i}$ which is maximal in the set $P_{1}, P_{2}, \cdots, P_{n}$. Thus $P$ is prime and hence a maximal prime of $A$. If conversely $P_{j}$ is maximal in $P_{1}, P_{2}, \cdots, P_{n}$, then $P_{j}$ must be a maximal nrp to $A$ ideal, for if not there exists $Q \supset P_{j}$ and such that $Q$ is nrp to $A$. But then by Lemma 5 we would have $Q$ contained in some $P_{i}$ and $P_{j} \subset Q \subseteq P_{i}$ contrary to the maximality of $P_{j}$.

LemMa 6. Let (1) be a reduced representation of $A$ by primal ideals with prime adjoints $P_{i}$. Then $A$ is primal if and only if one $P_{j}$ divides all the others, in which case $P_{j}$ is the adjoint of $A$.

Proof. (i) Let $P_{j} \supseteq P_{i}$ for all $i$, so that $P_{j}=P_{1} \cup P_{2} \cup \ldots \cup P_{n}$. Then by Lemma $5, B$ nrp to $A$ implies that $B \subseteq P_{j}$ and since by Lemma 5 again $P_{j}$ is nrp to $A$, it follows that $A$ is primal with adjoint $P_{j}$.

(ii) Let $A$ be primal with adjoint $P$. Then since $P$ is nrp to $A, P \subseteq P_{j}$ for some $j$ by Lemma 5 . Also by Lemma $5, P_{i}$ is nrp to $A$, hence $P_{i} \subseteq P$ for all $i$, or $P_{i} \subseteq P \subseteq P_{j}$ for all $i$. Then $P=P_{j}$ and the lemma is proved.

DEFINITION 12. If (1) is an irredundant representation of $A$ by primal ideals $A_{i}$, and is such that $A_{i} \cap A_{j}$ is not primal if $i \neq j$, it will be called a short representation of $A$ by primal ideals.

TheOREM 4. Let (1) be a reduced representation of $A$ by primal ideals with prime adjoints $P_{i}$. Then $A$ has a short representation by primal ideals whose adjoints are the maximal primes of $A$.

Proof. Since (1) is reduced we may assume that the representation is irredundant, since the intersection of any subset of $A_{1}, A_{2}, \cdots, A_{n}$ is also reduced. Let the indexing be such that $P_{1}, P_{2}, \cdots, P_{r}$ are the maximal elements of the set $P_{1}, P_{2}, \cdots, P_{n}$. Let $A_{1}^{\prime}$ denote the intersection of those 
$A_{i}$ such that $P_{i} \subseteq P_{1}$, and let $A_{j}^{\prime}$ denote the intersection of those $A_{i}$ such that $P_{i} \subseteq P_{j}$ but $P_{i} \Phi P_{k}$ if $k<j, j=2,3, \cdots, r$. Each of $A_{1}^{\prime}, A_{2}^{\prime}, \cdots, A_{r}^{\prime}$ satisfies the conditions of Lemma 6 and hence they are all primal with prime adjoints $P_{1}, P_{2}, \cdots, P_{r}$. Now for $j \neq k$, the intersection of the $A_{i}$ forming $A_{j}^{\prime}$ and $A_{k}^{\prime}$ is a reduced intersection of primal ideals not all of whose adjoints are contained in any one adjoint, hence by Lemma $6, A_{j}^{\prime} \cap A_{k}{ }_{k}^{\prime}$ is not primal and the representation $A=A_{1}^{\prime} \cap A_{2}^{\prime} \cap \cdots \cap A_{r}^{\prime}$ is short. By Theorem 3, $P_{1}$, $P_{2}, \cdots, P_{r}$ are the maximal primes of $A$, and the theorem is proved.

Corollary. If (1) is a representation of $A$ by strongly irreducible ideals, then $A$ has a short representation by primal ideals whose adjoints are the maximal primes of $A$.

Proof. By Lemma 3 each $A_{i}$ is primal with prime adjoint. We may assume the representation is irredundant. Since the $A_{i}$ are irreducible, Lemma II of $E$. Noether's paper [4], which remains valid in general rings, assures that the representation is reduced. The result now follows from the theorem.

THEOREM 5. In any short reduced representation of $A$ by primal ideals with prime adjoints, the adjoints and the number of primal components are uniquely determined.

Proof. Let $A=A_{1} \cap A_{2} \cap \cdots \cap A_{n}$, where $P_{i}$ is the adjoint of $A_{i}$ and $A=A_{1}^{\prime} \cap A_{2}^{\prime} \cap \cdots \cap A_{m}^{\prime}$, where $P_{j}^{\prime}$ is the adjoint of $A_{j}^{\prime}$ be any two such representations of $A$. Since both representations are short, no $P_{i}$ properly contains another $P_{j}$ and no $P_{i}^{\prime}$ properly contains another $P_{j}^{\prime}$. Then by Theorem 3 both $P_{1}, P_{2}, \cdots, P_{n}$ and $P_{1}^{\prime}, P_{2}^{\prime}, \cdots, P_{m}^{\prime}$ are the set of maximal primes of $A$, hence $m=n$ and the $P_{i}$ are the $P_{j}^{\prime}$ in some order.

By Lemma 1, every primal ideal in a uniform ring has a prime adjoint. Thus for uniform rings the results of this section are valid without the stipulation that the primal ideals in question have prime adjoints.

For later use we include here the following lemma.

Lemma 7. Let (1) be a reduced representation of $A$ by primal ideals such that the adjoint $Q_{i}$ of $A_{i}$ is unrp to $A_{i}$. Then an ideal is nrp to $A$ if and only if it is contained in some $Q_{i}$, and every ideal nrp to $A$ is unrp to $A$.

Proof. Since $Q_{1}$ is unrp to $A_{1}$, there exists $x \notin A_{1}$ such that $x R Q_{1} \subseteq A_{1}$. Let $A_{1}^{\prime}=(x)+A_{1}$, and we have $A_{1}^{\prime} \cap A_{2} \cap \cdots \cap A_{n} \supset A$ since (1) is reduced and $A_{1}^{\prime} \supset A_{1}$. Then there exists $y \notin A$ such that $y \in A_{1}^{\prime} \cap A_{2} \cap \cdots \cap A_{q_{n}}$. Now $y R Q_{1} \subseteq(x) R Q_{1}+A_{1} R Q_{1} \subseteq A_{1}$, and it follows that $y R Q_{1} \subseteq A$. Thus $Q_{1}$ is unrp to $A$, and similarly each $Q_{i}$ is unrp to $A$.

By Lemma 1 the $Q_{i}$ are all prime, and thus it follows from Lemma 5 that an ideal $B$ is nrp to $A$ if and only if $B$ is contained in some $Q_{i}$. But since each $Q_{i}$ is unrp to $A$ this implies that every ideal nrp to $A$ is unrp to $A$. 
4. Ascending chain condition. Throughout this section we shall assume that the ring $R$ satisfies the ascending chain condition for ideals.

Theorem 6. If $A$ is an irreducible ideal and $B$ is nrp to $A$, then $B$ is unrp to $A$.

Proof. The ascending chain condition implies that $B$ has a finite basis, or $B=\left(b_{1}, b_{2}, \cdots, b_{n}\right)$. Now $\left(A b_{1}^{-1} \cap A b_{2}^{-1} \cap \cdots \cap A b_{n}^{-1}\right) R B=A B^{-1} R B \subseteq A$. For each $i, A b_{i}^{-1} \supset A$, hence $A b_{1}^{-1} \cap A b_{2}^{-1} \cap \cdots \cap A b_{n}^{-1} \supseteq A$, and equality is impossible trivially if $n=1$, and if $n>1$ since $A$ is irreducible. Thus there exists $x \in A b_{1}^{-1} \cap A b_{2}^{-1} \cap \cdots \cap A b_{n}^{-1}$ such that $x \notin A$. For this $x$ we have $x R B \subseteq A$, and hence $B$ is unrp to $A$.

Theorem 7. Every ideal $A$ has a short reduced representation by primal ideals whose adjoints are the maximal primes of $A$ and are unrp to $A$.

Proof. The A.C.C. implies that $A$ has a finite representation, which we may assume to be irredundant, by irreducible ideals $A_{1}, A_{2}, \cdots, A_{n}$. By Lemma II of E. Noether's paper [4] which, together with Lemma IV of the same paper, remains valid in the noncommutative case, the representation is reduced. Then by Lemma 3 each $A_{i}$ is primal, hence by Theorem 6 the adjoint $P_{i}$ of $A_{i}$ is unrp to $A_{i}$. Then each $P_{i}$ is prime by Lemma 1 , and by Lemma 7 is unrp to $A$. Suppose $P_{1}, P_{2}, \cdots, P_{m}$ are the maximal elements of the inclusion ordered set $P_{1}, P_{2}, \cdots, P_{n}$. Let $A_{j}^{\prime}$ be the intersection of those $A_{i}$ whose adjoints $P_{i}$ are divisible by $P_{j}$ but not by $P_{k}$ for $k<j, j=1,2, \cdots, m$. By Lemma 6 each $A_{j}^{\prime}$ is primal. If we replace the $A_{i}$ composing $A_{1}^{\prime}$ by $A_{1}^{\prime}$, E. Noether's Lemma IV assures that the representation remains reduced, and similarly for $A_{2}^{\prime}, A_{3}^{\prime}, \cdots, A_{m}^{\prime}$. By Lemma 6 the resulting representation, $A=A_{1}^{\prime} \cap A_{2}^{\prime} \cap \cdots \cap A_{m}^{\prime}$, is short and by Theorem 3 the maximal primes of $A$ are exactly $P_{1}, P_{2}, \cdots, P_{m}$.

Corollary 1. Every ideal $A$ has a finite set of maximal primes, which are the maximal nrp to $A$ ideals.

Proof. The result is an immediate consequence of Theorem 7 and Theo. rem 3.

Corollary 2. If $A$ is primal the adjoint of $A$ is a prime ideal.

Theorem 8. Let $A=A_{1}^{\prime} \cap A_{2}^{\prime} \cap \cdots \cap A_{m}^{\prime}$ be the short reduced representation of Theorem 7, and $A_{i}=\left\{\cap B_{\alpha} \mid B_{\alpha} \supseteq A, B_{\alpha}\right.$ primal with adjoint $\left.Q_{\alpha} \subseteq P_{i}\right\}$. Then $A_{i}$ is primal with adjoint $P_{i}$ and is the minimal primal divisor of $A$ whose adjoint is contained in $P_{i}$. Also, $A=A_{1} \cap A_{2} \cap \cdots \cap A_{m}$ is a short representation of $A$.

Proof. (i) There exists $x_{i} \notin A_{i}^{\prime}$ such that $x_{i} R P_{i} \subseteq A_{i}^{\prime}$. Then there exists $x_{i}^{\prime} \notin A$ such that $x_{i}^{\prime} \in A_{1}^{\prime} \cap \cdots \cap A_{i-1}^{\prime} \cap\left(x_{i}, A_{i}^{\prime}\right) \cap A_{i+1}^{\prime} \cap \cdots \cap A_{m}^{\prime}$ since 
$A=A_{1}^{\prime} \cap A_{2}^{\prime} \cap \cdots \cap A_{m}^{\prime}$ is reduced. Thus $x_{i}^{\prime} \notin A_{i}^{\prime}, x_{i}^{\prime} \notin A_{i}$. But

$$
x_{i}^{\prime} R P_{i} \subseteq A_{1}^{\prime} \cap \cdots \cap A_{i-1}^{\prime} \cap\left[\left(x_{i}\right) R P_{i}+A_{i}^{\prime} R P_{i}\right] \cap A_{i+1}^{\prime} \cap \cdots \cap A_{m}^{\prime}=A \text {. }
$$

Hence $x_{i}^{\prime} R P_{i} \subseteq A_{i}$ while $x_{i}^{\prime} \notin A_{i}$ and $P_{i}$ is nrp to $A_{i}$. If $y$ is nrp to $A_{i}$, then $x R y \subseteq A_{i}$ for some $x \notin A_{i}$. Then $x \notin B$ for some $B \supseteq A, B$ primal with adjoint $Q \subseteq P_{i}$, and $x R y \subseteq B$ implies $y \in Q$, hence $y \in P_{i}$. Thus $P_{i}$ is the adjoint of $A_{i}$, and our first assertion is proved.

(ii) Clearly $A=A_{1} \cap A_{2} \cap \cdots \cap A_{m}$. If $m>1$, suppose $A=A_{2} \cap \cdots \cap A_{m}$. Now there exists $x \notin A$ such that $x R P_{1} \subseteq A$, hence $P_{1}$ is nrp to one of $A_{2}, A_{3}$, $\cdots, A_{m}$. Thus $P_{1}$ is divisible by one of $P_{2}, \cdots, P_{m}$, a contradiction, and the representation must be irredundant. If the representation is not short, suppose $A_{1} \cap A_{2}$ is primal with adjoint $P$. Then $x R P \subseteq A_{1} \cap A_{2}$ for some $x \notin A_{1} \cap A_{2}$. Say $x \notin A_{1}$. Then $P$ is nrp to $A_{1}$, hence $P \subseteq P_{1}$ and we have $A_{1} \cap A_{2} \supseteq A_{1}$ by definition of $A_{1}$. But this implies $A_{1}=A_{1} \cap A_{2}$ and the representation is redundant, which is impossible.

THEOREM 9. Every ideal is the intersection of its upper isolated components $U\left(A, P_{1}\right), U\left(A, P_{2}\right), \cdots, U\left(A, P_{n}\right)$ where $P_{1}, P_{2}, \cdots, P_{n}$ are the maximal primes of $A$.

Proof. By Theorem 7, $A$ has a representation $A=A_{1} \cap A_{2} \cap \cdots \cap A_{n}$ where $A_{i}$ is primal with adjoint $P_{i}$. As in the proof of Theorem 2, we have $A \subseteq U\left(A, P_{i}\right) \subseteq U\left(A_{i}, P_{i}\right)=A_{i}$. Hence it follows that $A \subseteq \bigcap_{i=1}^{n} U\left(A, P_{i}\right)$ $\subseteq \bigcap_{i=1}^{n} A_{i}=A$, and the theorem is proved.

Theorem 10. Any ring satisfying the A.C.C. for ideals is a uniform ring.

Proof. If $A$ is an ideal in a ring with A.C.C., let $B$ be any ideal nrp to $A$. Then $B$ is contained in a maximal nrp to $A$ ideal $P$ which by Theorem 7 , Corollary 1 is a maximal prime of $A$, and by Theorem 7 is unrp to $A$. Thus there exists $x \notin A$ such that $x R P \subseteq A$, hence $x R B \subseteq x R P \subseteq A$ and $B$ is unrp to A.

That a uniform ring need not satisfy the A.C.C. can be shown by the following example.

Let $F$ be a field with a valuation $\Phi$ such that the value group of $F$ is the rational numbers. Let $R$ be the ring of all $f \in F$ for which $\Phi(f) \geqq 1$. It may be readily shown that if $A$ is a proper ideal in $R$ and $B$ is any ideal of $R$ we can always find $x \in R$ such that $x \notin A$ while $x B \subseteq A$, hence $x R B \subseteq A$ and $B$ is unrp to $A$. But the A.C.C. clearly does not hold in $R$. We may remark that this is an example of a ring in which every proper ideal is primal with adjoint $R$.

Murdoch [6] has defined an ideal $Q$ to be right primary if every element not in the McCoy radical [5] of $Q$ is $\operatorname{rp}$ to $Q$.

LEMMA 8. If $Q$ is right primary with radical $P$, then $Q$ is primal with prime adjoint $P$. 
Proof. By a result of Murdoch [6] $P$ is nrp to $Q$. By definition all elements not in $P$ are rp to $Q$, hence the set of all elements nrp to $Q$ is exactly $P$ and $Q$ is primal with adjoint $P$. By Corollary 2, Theorem 7, $P$ is prime.

TheOREM 11. Let $A=Q_{1} \cap Q_{2} \cap \cdots \cap Q_{n}$ be an irredundant representation of $A$ by right primary ideals with radicals $P_{1}, P_{2}, \cdots, P_{n}$. Then the maximal elements of the set $P_{1}, P_{2}, \cdots, P_{n}$ are the maximal primes of $A$.

Proof. Murdoch has shown in [6] that an element $x$ is rp to $A$ if and only if $x$ is in the complement of every $P_{i}$. Hence each $P_{i}$ is nrp to $A$ and if $B$ is nrp to $A$ then $B \subseteq P_{1} \cup P_{2} \cup \cdots \cup P_{n}$. By Lemma 8 each $P_{i}$ is prime. We now repeat the argument used in part (i) of the proof of Lemma 5 , and obtain $B \subseteq P_{i}$ for some $i$. The argument now proceeds exactly as in the proof of Theorem 3.

5. Maximal primes and associated primes. If a minimal prime ideal of $A$ is defined to be an ideal which is minimal in the inclusion ordered set of prime divisors of $A$, then the intersection of the minimal primes of $A$ has been shown in [5] to be the McCoy radical of $A$.

For commutative rings Fuchs has characterized in [3] the intersection of the maximal primes of an ideal. In the case of ideals which possess reduced representations by primal ideals with prime adjoints it is possible to extend Fuchs' result to noncommutative rings. As we have seen, this condition is satisfied for any ideal in a ring with A.C.C.

Definition 13. If $A$ is any ideal in a general ring, the adjoint ideal of $A$ is defined to be the set of all $x$ such that $(x, y)$ is nrp to $A$ whenever $y$ is nrp to $A$. That this set does form an ideal is easily shown. In virtue of the fact that if $R$ is commutative then $x \operatorname{nrp}$ to $A$ is equivalent to $x$ not prime to $A$, we see that the above definition is the same as Fuchs' [3] in the commutative case.

We note that $A$ is trivially contained in the adjoint of $A$. In the event that $A$ is primal, then the adjoint $Q$ of $A$ defined previously coincides with the adjoint $Q^{\prime}$ of Definition 13 .

THEOREM 12. The adjoint ideal $Q^{\prime}$ of $A$ is the intersection of the maximal nrp to $A$ divisors of $A$.

Proof. Suppose $x \in Q^{\prime}$ and $B$ is any ideal nrp to $A$. Then $(x)+B$ is nrp to $A$ since $y \in(x)+B$ implies $y \in(x, b)$ for some $b \in B$ and thus $y$ is nrp to $A$ by the definition of $Q^{\prime}$. Hence $Q^{\prime}$ is contained in every maximal nrp to $A$ ideal. Conversely, let $x$ be in every maximal nrp to $A$ ideal and $y$ be nrp to $A$. Then $y$ is in some maximal nrp to $A$ ideal $B,(x, y) \subseteq B$ and $(x, y)$ is nrp to $A$ so that $x \in Q^{\prime}$ and the theorem is proved.

THEOREM 13. If $A$ has a reduced representation by primal ideals with prime adjoints, then the adjoint of $A$ is the intersection of the maximal primes of $A$. 
Proof. By Theorem 3 the maximal nrp to $A$ ideals are precisely the maximal primes of $A$, namely the maximal elements of the set of adjoint primes of the primal ideals in the given representation of $A$. The result now follows at once from Theorem 12 .

McCoy [5] has noted that any prime divisor of $A$ contains a minimal prime of $A$. Thus it follows that the adjoint of an ideal possessing a reduced representation by primal ideals with prime adjoints always contains the McCoy radical of $A$.

We turn now to a consideration of the prime ideals "associated" with a given ideal. Such ideals have been defined by Krull [4] for noncommutative rings. Since our point of view is considerably different from that of Krull, however, we shall give a new definition which is derived from the method of Murdoch [6]. He has shown that if an ideal $A$ in a ring with A.C.C. has a short representation by right primary ideals with radicals $P_{1}, P_{2}, \cdots, P_{n}$, then $P_{i}$ is nrp to $A$, and a prime $P$ which divides $A$ is nrp to $U(A, P)$ if and only if $P$ is one of $P_{1}, P_{2}, \cdots, P_{n}$.

Definition 14. A prime ideal $P$ containing $A$ is a (right) associated prime of $A$ if $P$ is nrp to $A$ and also nrp to $U(A, P)$.

Thus in a ring with A.C.C., if an ideal $A$ has a short representation by right primary ideals, their radicals are exactly the associated primes of $A$.

Lemma 9. Let $A$ be an ideal with a short reduced representation by primal ideals $A_{1}, A_{2}, \cdots, A_{n}$ whose adjoints $P_{1}, P_{2}, \cdots, P_{n}$ are such that $P_{i}$ is unrp to $A_{i}$. Then $P_{i}$ is unrp to $U\left(A, P_{i}\right)$ for $i=1,2, \cdots, n$.

Proof. By Lemma 1 , each $P_{i}$ is a prime ideal. By Lemma 7 each $P_{i}$ is unrp to $A$. Hence there exists $x_{i} \notin A$ such that $x_{i} R P_{i} \subseteq A$ and thus $x_{i} R P_{i}$ $\subseteq U\left(A, P_{j}\right)$ for every $j$. Now $x_{i} \notin A$ implies $x_{i} \notin A_{j}$ for some $j$. Then $x_{i} R P_{i}$ $\subseteq A_{j}$ implies $P_{i} \subseteq P_{j}$ but since $P_{i}$ and $P_{j}$ are both maximal this implies $P_{i}=P_{j}$ and $i=j$. From $A_{i}=U\left(A_{i}, P_{i}\right) \supseteq U\left(A, P_{i}\right)$ it follows that $x_{i} \notin U\left(A, P_{i}\right)$ and $P_{i}$ is unrp to $U\left(A, P_{i}\right)$.

THEOREM 14. In a uniform ring, if an ideal $A$ has a short reduced representation by primal ideals with adjoints $P_{1}, P_{2}, \cdots, P_{n}$, then a prime ideal $P$ is a maximal prime of $A$ if and only if $P$ is a maximal element in the inclusion ordered set of associated primes of $A$.

Proof. By Lemma 9 each $P_{i}$ is an associated prime of $A$, and by Theorem 3 the $P_{i}$ are the maximal nrp to $A$ ideals, hence the maximal primes of $A$. Clearly every associated prime of $A$ is contained in a maximal nrp to $A$ ideal, and hence in some $P_{i}$. Thus if $P$ is a maximal associated prime of $A$ it must be one of the $P_{i}$, hence a maximal prime of $A$. Conversely, every maximal prime of $A$ is one of the $P_{i}$ and hence a maximal associated prime of $A$.

Corollary. If $A$ is an ideal in a ring with the A.C.C. for ideals, then the maximal primes of $A$ are the maximal associated primes of $A$. 
Proof. By Theorem 7 every ideal $A$ has a short reduced representation by primal ideals, and by Theorem $10, R$ is a uniform ring. The result now follows at once from Theorem 14 .

We note that by the corollary the representations of an ideal in a ring with A.C.C. obtained in Theorems 8 and 9 are identical. For if $P$ is a maximal prime of $A$, then $U(A, P)$ is primal with adjoint $P$ and by definition is contained in every primal divisor of $A$ whose adjoint is contained in $P$, hence is the minimal such divisor of $A$.

As we noted in $\S 1$, if $R$ is a uniform ring with unit element then the definition of $B \mathrm{nrp}$ to $A$ is equivalent to that of Curtis [1]. Hence the respective definitions of the maximal primes of $A$ are also equivalent in such a ring. For a ring with unit element satisfying the A.C.C. for ideals, Curtis defined the (right) isolated $B$-component ideal of $A$ to be the ideal $I(A, B)=A B^{-q}$ for $q \geqq 0$ and such that $A B^{-q}=A B^{-q-1}$, and a (right) associated prime ideal of $A$ to be a prime ideal $P$ such that $I(A, P) \supset A$ and $[I(A, P)]^{-1} A \subseteq P$ where $[I(A, P)]^{-1} A=\left\{\sum C \mid I(A, P) C \subseteq A\right\}$. He then proved that the maximal primes of $A$ are the maximal elements of the inclusion ordered set of (right) associated prime ideals of $A$. Thus we see that for a ring with unit element and A.C.C. for ideals, the two definitions of associated prime ideals of an ideal $A$ both lead to the same set of maximal associated prime ideals.

6. Isolated components of an ideal. McCoy has defined in [5] an $m$-system to be a set $M$ of elements of $R$ with the property that if $x$ and $y$ are in $M$ then there exists $r$ in $R$ such that $x r y$ is in $M$. The null set is also considered an $m$-system. Thus an ideal is prime if and only if its complement is an $m$-system. Murdoch has defined [6] a right $M$-n-system to be a set $N$ containing an $m$-system $M$ and with the property that for given $m$ in $M$ and $n$ in $N$ there exists $r$ in $R$ such that $n r m$ is in $N$. If $M$ is the null set then the only $M$-n-system is $M$ itself. He then defined the (right) upper isolated $M$-component of an ideal $A$ not intersecting $M$ to be the set of elements $x$ such that every right $M$-n-system containing $x$ also contains an element of $A$. We shall adopt these definitions with the exception that if $M$ is the null set then any set in $R$ is a right $M$-n-system, a change which results in our Definition 10 of the (right) isolated $P$-component of $A$ where $P$ is a prime divisor of $A$. We now define the (right) upper isolated $B$-component, $U(A, B)$, where $B$ is any ideal divisor of $A$. We shall call a set $M$ entirely rp (erp) to $A$ if every element in $M$ is rp to $A$.

Definition 15. If $B \supseteq A$, then the (right) upper isolated $B$-component of $A$, $U(A, B)$, is the intersection of all ideal divisors of $A$ to which the set $M$ of elements rp to $B$ is erp.

Definition 16. Let $B$ be a (proper) ideal in $R$ and $M$ be the set of elements in $R$ which are rp to $B$. If $M$ is non-null a set $N$ containing $M$ is a (right) $B$ - $\nu$-system if for every $m \in M$ and $n \in N$ there exists some $r \in R$ such that $n r m \in N$. If $M$ is null ( $R$ is nrp to $B$ ) then any set is a $B$ - $\nu$-system. Similarly, any set is considered to be an $R$ - $\nu$-system. 
We note that $C(B)$, the complement in $R$ of $B$, is a $B-\nu$-system. For if $M$ is non-null then $m \in M$ and $x R m \subseteq B$ implies $x \in B$, hence $m \in M$ and $x \in C(B)$ implies the existence of some $r \in R$ such that $\operatorname{xrm} \in C(B)$, while if $M$ is null then $C(B)$ is a $B$ - $\nu$-system by definition.

Lemma 10. Let $N$ be any $B$ - $\nu$-system disjoint from $A$. Then $A$ is contained in a maximal ideal $Q$ disjoint from $N$ and $M$ is erp to $Q$.

Proof. Since the union of any ascending chain of ideals containing $A$ and disjoint from $N$ is again an ideal containing $A$ and disjoint from $N$ the existence of $Q$ follows at once from Zorn's lemma. If $y \notin Q$ then, by the maximality of $Q$, there exists $n \in N$ such that $n \in(y)+Q$. Then $n$ has the form $n=i y+r y$ $+y r^{\prime}+\sum_{i j} r_{i} y r_{j}+q$, for $r, r^{\prime}, r_{i}, r_{j}$ all in $R$ and $q$ in $Q$. Now if $m$ is rp to $B$ there exists $x \in R$ such that $n x m \in N$, or $n x m=i y x m+r y x m+y r^{\prime} x m$ $+\sum_{i j} r_{i} y r_{j} x m+q x m$ is in $N$. But if $y R m \subseteq Q$ then $n x m \in Q \cap N$, contradicting $Q$ disjoint from $N$. Hence $y R m \subseteq Q$ implies $y \in Q$ and $m$ is rp to $Q$ as required.

THEOREM 15. The complement in $R$ of $U(A, B)$ is the maximal $B$ - $\nu$-system aisjoint from $A$, and $U(A, B)$ itself is the set consisting of all $x$ such that every $B$ - $\nu$-system containing $x$ intersects $A$.

Proof. Let $N$ be the complement of $U(A, B)$. Then $x \in N$ implies that $x \notin Q$ for some ideal $Q \supseteq A$ and such that $M$ is erp to $Q$. Hence for $m \in M$ there exists $r \in R$ such that $x r m \in N$. Thus $N$ is a $B$ - $\nu$-system, and is trivially disjoint from $A$. Suppose $N^{\prime}$ is any $B$ - $\nu$-system which is disjoint from $A$. Then by Lemma 10 we have $A \subseteq Q^{\prime}$ for $Q^{\prime}$ an ideal disjoint from $N^{\prime}$ and such that $M$ is erp to $Q^{\prime}$. But then $U(A, B) \subseteq Q^{\prime}$ by definition, hence $N \supseteq C\left(Q^{\prime}\right)$ $\supseteq N^{\prime}$. Thus $N$ is the maximal $B$ - $\nu$-system disjoint from $A$. The second assertion follows at once from the first, and the theorem is proved.

Corollary. If $B \supseteq A \supseteq A^{\prime}$, then $U(A, B) \supseteq U\left(A^{\prime}, B\right)$.

Proof. Every ideal $J$ containing $A$ to which $M$ is erp also contains $A^{\prime}$, and hence the intersection of all such ideals containing $A$ contains the intersection of all such ideals containing $A^{\prime}$.

We may remark that the last property in Theorem 15 could have been used to define $U(A, B)$, and the others derived therefrom. Such a method would have been more conventional, the proof proceeding by way of the standard three lemmas (cf. e.g. McCoy [5] or Murdoch [6]), our versions of which would have read as follows: (i) if $R \supseteq B \supseteq A$ then there exists a unique maximal $B$ - $\nu$-system disjoint from $A$, (ii) same as Lemma 10 , and (iii) a set $Q$ is a minimal ideal dividing $A$ such that $M$ is erp to $Q$ if and only if $C(Q)$ is a maximal $B$ - $\nu$-system disjoint from $A$.

In the event $P$ is a prime ideal divisor of $A$, then the ideal $U(A, P)$ is the upper isolated $P$-component of $A$ of Definition 12, as in this case the set of elements rp to $P$ is just the complement of $P$. 
Murdoch has defined in [6] the right lower isolated component of an ideal $A$, relative to an $m$-system $M$ disjoint from $A$, to be the set of all elements $x$ of $R$ such that $x R m \subseteq A$ for some $m$ of $M$. Thus if $P$ is a prime divisor of $A$ the right lower isolated component of $A$ relative to $P$ is the set $\{x \mid x R m \subseteq A$ for some $m \in P\}$. We now extend this concept to the case of $B$ any ideal divisor of $A$, and establish relations between the upper and lower isolated $B$ components of $A$ corresponding to those obtained by Murdoch.

Definition 17. If $B$ is an ideal containing $A$, let $M$ be the set of all $m$ in $R$ which are rp to $B$. If $M$ is non-null the (right) lower isolated $B$-component of $A$ is the ideal $L(A, B)=\left\{\sum A m^{-1} \mid m \in M\right\}$. If $M$ is null then $L(A, B)$ is $A$, and $L(A, R)$ is also $A$.

We note that if $P$ is a prime ideal different from $R$ this definition agrees with that of Murdoch, since then the set $M$ is an $m$-system.

Lemma 11. If $R \supseteq B \supseteq A$ then $U(A, B) \supseteq L(A, B) \supseteq A$.

Proof. By definition $L(A, R)=A=U(A, R)$, and if $M$ is null then $L(A, B)$ $=A=U(A, B)$ also by definition.

Suppose $M$ is non-null and $R \neq B$. That $L(A, B) \supseteq A$ follows at once from the fact that $A m^{-1} \supseteq A$ for any $m \in M$. If $x \in L(A, B)$ then $x=\sum_{i=1}^{n} x_{i}$ for $x_{i} \in A m_{i}^{-1}, m_{i} \in M$. Now $x_{i} \in A m_{i}^{-1}$ implies $x_{i} R m_{i} \subseteq A$ and every $B$ - $\nu$-system containing $x_{i}$ certainly contains an element of $A$, hence $x_{i} \in U(A, B)$ for $i=1,2, \cdots, n$. Thus $x=\sum_{i=1}^{n} x_{i}$ is in $U(A, B)$ and $L(A, B) \subseteq U(A, B)$.

THEOREM 16.
(a) $U(U(A, B), B)=U(A, B)$,
(b) $L(U(A, B), B)=U(A, B)$,
(c) $U(L(A, B), B)=U(A, B)$.

Proof. (a) The complement in $R$ of $U(A, B)$ is a $B$ - $\nu$-system $N$ not intersecting $U(A, B)$ by Theorem 15 . Hence it is certainly the maximal such system, and it follows from the same theorem that $C(N)=U(A, B)$ is the upper isolated $B$-component of $U(A, B)$.

(b) By (a) just proved and Lemma 11 we obtain

$$
L(U(A, B), B) \subseteq U(U(A, B), B)=U(A, B) \subseteq L(U(A, B), B)
$$

and the desired equality follows.

(c) Since $L(A, B) \subseteq U(A, B)$ we may apply the corollary to Theorem 15 to obtain $U(L(A, B), B) \subseteq U(U(A, B), B)$. Since $A \subseteq L(A, B)$ we apply the same corollary to obtain $U(A, B) \subseteq U(L(A, B), B)$. Combining these results with part (a) we have $U(L(A, B), B) \subseteq U(U(A, B), B)=U(A, B) \subseteq U(L(A$, $B), B$ ) and the equality follows.

Definition 18. For all ordinal numbers $\alpha$ we define $L^{\alpha}(A, B)$ by induction as follows: $L^{1}(A, B)=L(A, B)$. If $\alpha$ is not a limit ordinal then $L^{\alpha}(A, B)$ 
$=L\left(L^{\alpha-1}(A, B), B\right)$, and if $\alpha$ is a limit ordinal then $L^{\alpha}(A, B)$ is the union of all $L^{\beta}(A, B)$ for $\beta<\alpha$.

Evidently if $\beta<\alpha$ then $L^{\beta}(A, B) \subseteq L^{\alpha}(A, B)$.

Theorem 17. For all ordinal numbers $\alpha, U(A, B) \supseteq L^{\alpha}(A, B)$.

Proof. For $\alpha=1$ the result is known by Lemma 11 . We assume the result for all ordinals less than $\alpha$ and proceed by induction.

(i) If $\alpha$ is not a limit ordinal and so has an immediate predecessor $\alpha-1$ then we have

$$
\begin{aligned}
L^{\alpha}(A, B) & =L\left(L^{\alpha-1}(A, B), B\right) & & \text { by definition, } \\
& \subseteq U\left(L^{\alpha-1}(A, B), B\right) & & \text { by Lemma } 11, \\
& \subseteq U(U(A, B), B) & & \text { by Theorem 15, Corollary, } \\
& =U(A, B) & & \text { by Theorem 16(a). }
\end{aligned}
$$

(ii) If $\alpha$ is a limit ordinal, then by the definition of $L^{\alpha}(A, B)$ we have that $x \in L^{\alpha}(A, B)$ implies $x \in L^{\beta}(A, B)$ for some $\beta<\alpha$. But then by the inductive assumption $x \in U(A, B)$ and hence $L^{\alpha}(A, B) \subseteq U(A, B)$.

THEOREM 18. For any ordinal number $\alpha, L^{\alpha}(A, B)=L^{\alpha+1}(A, B)$ if and only if $L^{\alpha}(A, B)=U(A, B)$.

Proof. (i) If $L^{\alpha}(A, B)=U(A, B)$ then by Theorem 16(b) we have $L^{\alpha+1}(A, B)$ $=L(U(A, B), B)=U(A, B)$.

(ii) Suppose $L^{\alpha}(A, B)=L^{\alpha+1}(A, B)$ for some $\alpha$, and $x R m \subseteq L^{\alpha}(A, B)$ for some $m \mathrm{rp}$ to $B$. (If $M$ is null, the result is trivial, as then all the component ideals are just $A$.) Then $x \in L^{\alpha}(A, B) m^{-1} \subseteq L^{\alpha+1}(A, B)=L^{\alpha}(A, B)$ and $m$ is rp to $L^{\alpha}(A, B)$ if $m$ is rp to $B$. But $U(A, B)$ is the minimal ideal containing $A$ to which every $m$ rp to $B$ is $\mathrm{rp}$, hence $U(A, B) \subseteq L^{\alpha}(A, B)$. Since $L^{\alpha}(A, B)$ $\subseteq U(A, B)$ for all $\alpha$, the result follows.

Corollary 1. There exists an ordinal $\alpha$, finite or transfinite, such that $L^{\alpha}(A, B)=U(A, B)$.

Proof. Under inclusion the $L^{\sigma}(A, B)$ form a bounded, well ordered set such that the union of any subset is again an $L^{\sigma}(A, B)$. Hence there exists a maximal element $L^{\alpha}(A, B)$ which must contain all $L^{\sigma}(A, B)$, thus $L^{\alpha}(A, B)$ $=L^{\alpha+1}(A, B)=U(A, B)$.

Corollary 2. If the A.C.C. for ideals holds in $R / A$ then $L^{n}(A, B)=U(A, B)$ for some finite $n$.

In the event $R$ is a commutative ring, then the set of elements rp to an ideal $B$ is the set of elements prime to $B$, which forms a multiplicative system. Thus both $U(A, B)$ and $L(A, B)$ as we have defined them are the same 
as the components defined by Murdoch in [6], which he has shown to be both equivalent to Krull's isolated component in the commutative case.

7. Principal components of an ideal. In Theorem 2 we saw that any ideal $A$ is the intersection of its upper isolated $P_{\alpha}$-components, where the $P_{\alpha}$ are the prime adjoints of the strongly irreducible primal divisors of $A$. If the ring $R$ satisfies the A.C.C. for ideals, Theorem 9 shows that $A$ is the finite intersection of its upper isolated components corresponding to the maximal primes of $A$. Since for any divisor $B$ of $A$ we have $A \subseteq L(A, B) \subseteq U(A, B)$, Theorems 2 and 9 remain valid if we replace "upper" by "lower." Moreover, we can then obtain a result for general rings similar to the modified version of Theorem 9.

Definition 19. If $R \supseteq Q$ and $Q$ is a maximal nrp to $A$ ideal, then $L(A, Q)$ is a (right) lower principal component of $A$, and $U(A, Q)$ is a (right) upper principal component of $A$.

We note that if $R$ is commutative, these definitions are equivalent to that of Krull's principal component.

ThEOREM 19. In any ring $R$, any ideal $A$ is the intersection of its lower principal components.

Proof. Let $\left\{Q_{\alpha}\right\}$ be the set of maximal nrp to $A$ ideals.

(i) If $R=Q_{\alpha}$ for some $\alpha$, then $L\left(A, Q_{\alpha}\right)=A$ by definition, or if $R$ is nrp to $Q_{\alpha}$ for some $\alpha$, then $L\left(A, Q_{\alpha}\right)=A$ by definition, and since $A \subseteq \bigcap L\left(A, Q_{\alpha}\right)$ the result follows trivially in either of these cases.

(ii) Suppose $R \neq Q_{\alpha}$ and $R$ is rp to $Q_{\alpha}$ for all $\alpha$, and suppose $x \in \cap L\left(A, Q_{\alpha}\right)$. Then if $Q$ is any $Q_{\alpha}$, we have $x=\sum_{i=1}^{n} x_{i}$ where there exists $m_{i}$ rp to $Q$ such that $x_{i} R m_{i} \subseteq A, i=1,2, \cdots, n$. Let $B=\{y \mid x R y \subseteq A\}$. Clearly $B$ is an ideal. Now we have $x R\left(m_{1} R m_{2} \cdots m_{n-1} R m_{n}\right)=\left(\sum_{i=1}^{n} x_{i}\right) R\left(m_{1} R m_{2} \cdots m_{n-1} R m_{n}\right)$ $\subseteq A$, hence $m_{1} R m_{2} \cdots m_{n-1} R m_{n} \subseteq B$.

Now if $B \subseteq Q$, then $\left(m_{1} R m_{2} \cdots m_{n-1}\right) R m_{n} \subseteq Q$ and $m_{n}$ rp to $Q$ implies $\left(m_{1} R m_{2} \cdots m_{n-2}\right) R m_{n-1} \subseteq Q$. Continuing this process, we find $m_{1} \in Q$, a contradiction. Hence $B \Phi Q_{\alpha}$ for any $\alpha$, and $B$ must be rp to $A$. But then there exists $b \in B$ such that $y R b \subseteq A$ implies $y \in A$. Now $x R b \subseteq A$ by definition of $B$, hence $x \in A$, and we have shown $\cap L\left(A, Q_{\alpha}\right) \subseteq A$. But $A \subseteq \cap L\left(A, Q_{\alpha}\right)$ trivially and the result follows.

It appears unlikely that a similar result holds in general for the upper principal components of an ideal. However, under some circumstances such a result can be obtained.

Theorem 20. If the adjoint $P_{\alpha}$ of a strongly irreducible divisor $A_{\alpha}$ of $A$ is rp to $A$, then $U\left(A, P_{\alpha}\right)$ is redundant in $A=\bigcap U\left(A, P_{\alpha}\right)$.

Proof. We recall that by Theorem $1, A=\cap A_{\alpha}$, where $\left\{A_{\alpha}\right\}$ is the set of strongly irreducible divisors of $A$, and by Theorem 2, $A=\cap U\left(A, P_{\alpha}\right)$, where 
$P_{\alpha}$ is the adjoint of $A_{\alpha}$. Now if $U\left(A, P_{\alpha}\right)$ is irredundant, then $A_{\alpha}$ is also. Hence $A_{\alpha} \supseteq \cap_{\beta \neq \alpha} A_{\beta}$ and $A_{\alpha}+\cap_{\beta \neq \alpha} A_{\beta} \supset A_{\alpha}$. Since, by Lemma $2, P_{\alpha}$ is unrp to $A_{\alpha}$, there exists $y \notin A_{\alpha}$ such that $y R P_{\alpha} \subseteq A_{\alpha}$. Let $A_{\alpha}^{\prime}=(y)+A_{\alpha} \supset A_{\alpha}$ and we have $A_{\alpha}^{\prime} \cap\left[A_{\alpha}+\bigcap_{\beta \neq \alpha} A_{\beta}\right]=A_{\alpha}+A_{\alpha}^{\prime} \cap\left(\bigcap_{\beta \neq \alpha} A_{\beta}\right) \supset A_{\alpha}$ by Dedekind's law and the fact that $A_{\alpha}$ is irreducible. Hence there exists $x \notin A_{\alpha}$ such that $x \in A_{\alpha}+(y)$ and $x \in A_{\beta}$ for $\beta \neq \alpha$. Now $x=a_{\alpha}+y^{\prime}$ for some $a_{\alpha} \in A_{\alpha}$ and $y^{\prime} \in(y)$, hence $x R P_{\alpha} \subseteq a_{\alpha} R P_{\alpha}+y^{\prime} R P_{\alpha} \subseteq A_{\alpha}$. But $x R P_{\alpha} \subseteq A_{\beta}$ for all $\beta \neq \alpha$, hence $x R P_{\alpha} \subseteq A$ and $P_{\alpha}$ is unrp to $A$. Thus if $P_{\alpha}$ is rp to $A$, then $U\left(A, P_{\alpha}\right)$ must be redundant.

Theorem 21. If in $A=\cap U\left(A, P_{\alpha}\right)$, where the $P_{\alpha}$ are the adjoints of the strongly irreducible divisors of $A$, all the redundant components can be eliminated, then $A$ is the intersection of its upper principal components.

Proof. If all the redundant components can be eliminated (certainly any finite number can), then by Theorem 20, the remaining ones have $P_{\alpha}$ unrp to $A$. Now each of these $P_{\alpha}$ is contained in a maximal nrp to $A$ ideal $Q_{\alpha}$. If $Q_{\alpha}=R$, then $U\left(A, Q_{\alpha}\right)=A \subseteq U\left(A, P_{\alpha}\right)$ by definition. If $Q_{\alpha} \neq R$ let $M=\{x \mid x$ rp to $\left.Q_{\alpha}\right\}$. Then $M \subseteq C\left(P_{\alpha}\right)$, and if $C\left(P_{\alpha}\right)$ is erp to an ideal $B \supseteq A$, so is $M$. Hence $U\left(A, P_{\alpha}\right) \supseteq U\left(A, Q_{\alpha}\right) \supseteq A$ in this case also. Thus $A=\cap U\left(A, Q_{\alpha}\right)$, the intersection of the upper principal components of $A$.

\section{REFERENCES}

1. C. W. Curtis, On additive ideal theory in general rings, Amer. J. Math. vol. 76 (1952) pp. 687-700.

2. L. Fuchs, On primal ideals, Proc. Amer. Math. Soc. vol. 1 (1950) pp. 1-8.

3. - On a special property of the principal components of an ideal, Det Kongelige Norske Videnskabers Selskab vol. 22 (1950) pp. 28-30.

4. W. Krull, Zweiseitige Ideale in nichtkommutativen Bereichen, Math. Zeit. vol. 28 (1928) pp. $481-503$.

5. N. H. McCoy, Prime ideals in general rings, Amer. J. Math. vol. 71 (1948) pp. 823-833.

6. D. C. Murdoch, Contributions to noncommutative ideal theory, Canadian Journal of Mathematics vol. 4 (1952) pp. 43-57.

7. E. Noether, Idealtheorie in Ringbereichen, Math. Ann. vol. 83 (1921) pp. 24-66.

University of British Columbia, VANCOUVER, B. C.

State College of Washington, Pullman, Wash. 